\section{Rotatory Dispersion of Cytochrome c}

The iron atom in hæm compounds has its six covalences directed toward the six corners of an octahedron; there are four co-planar bonds with the porphyrin nitrogen atoms and one bond on each side of the plane of the porphyrin. The only possible plane of symmetry in such molecules is the plane of the porphyrin; the iron must constitute a centre of asymmetry if one of two requirements is satisfied : (1) different groups are linked to iron on the two sides of the porphyrin plane or (2) groups co-ordinated to iron are also attached to porphyrin side-chains, thus forming chelate rings.

Both these conditions are fulfilled in cytochrome $c$, since the amino-acid composition differs on the two sides of the porphyrin ${ }^{1}$, and since the protein is linked to both iron ${ }^{2}$ and to porphyrin side-chain ${ }^{3}$. The iron atom of cytochrome $c$ should, therefore, contribute to the optical activity of the molecule, unless it is racemized, and racemization is not expected in biological compounds.

It has been observed that the oxidized and reduced forms of tris-orthophenanthroline-ruthenium exhibit marked differences in optical rotation, with no change in the organic portion of the molecule ${ }^{4}$. Optical activity due to iron in cytochrome $c$ may thus be differentiated from the optical activity due to the protein by looking for a difference in rotation resulting from the gain or loss of an electron.

Sigma cytochrome $c$ was purified by passing it through a column of $X R-64$ resin ${ }^{5}$. A $0 \cdot 5 M$ solution in phosphate buffer $(p \mathbf{H} 6 \cdot 5)$ was oxidized with

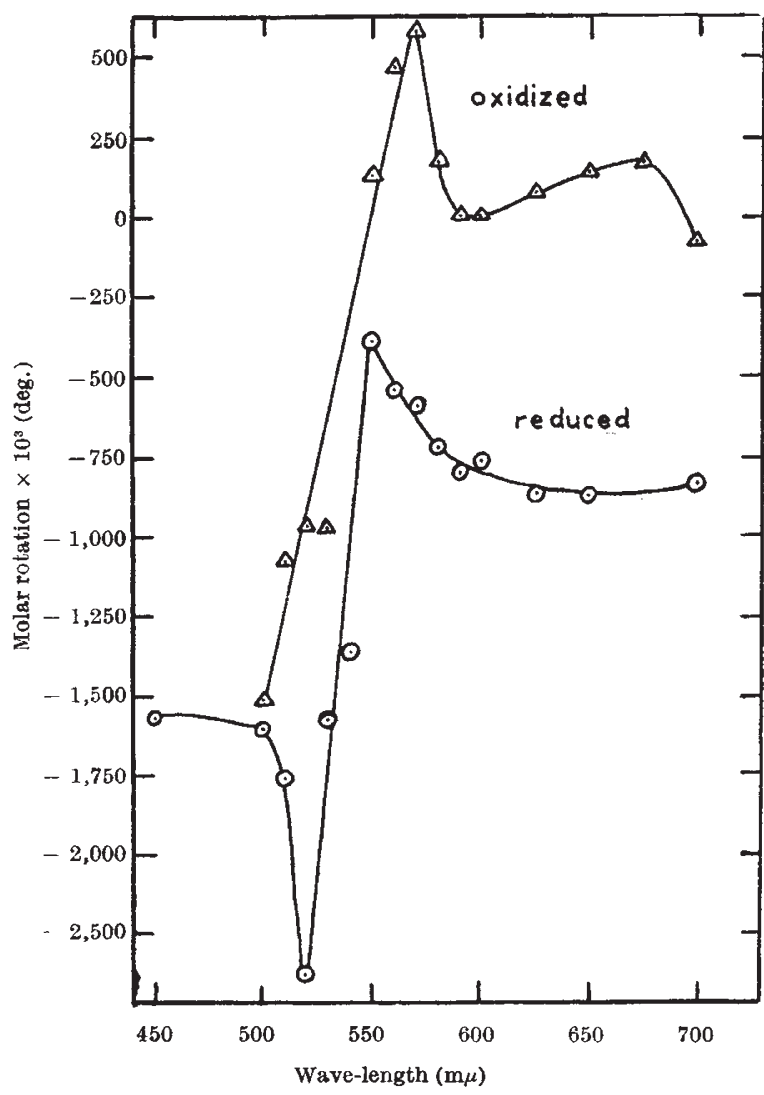

Fjg. 1. Rotatory dispersion curves for cytochrome c. (S.D. \pm $50 \times 10^{-3} \mathrm{deg}$.) potassium ferricyanide, reduced with hydrogen over platinum, and re-oxidized with ferricyanide. Optical measurements were made in a $0.5 \mathrm{~cm}$. cell at $26^{\circ}$ with a Rudolf polarimeter, using a zirconium light source and a Beckman monochromator. Fig. 1 shows rotatory dispersion curves for the oxidized and reduced forms; the curve obtained by oxidation of the reduced form is identical with the curve for the solution prior to reduction.

Both oxidized and reduced cytochrome $c$ show 'anomalous' dispersion in the region of $550 \mathrm{~m} \mu$, where the greatest difference exists in the spectra of the two forms ${ }^{6}$. The absorption in this region probably involves the absorption of the iron-nitrogen bonds; the 'anomalous' dispersion is another indication that the iron is asymmetric.

Similar investigations are being made for other biological porphyrin compounds.

This work was supported by a grant from the National Heart Institute, National Institutes of Health, Public Health Service. Use of the facilities of the Naval Medical Research Institute, Bethesda, Md., and of the polarimeter of the Laboratory of Natural Products, National Heart Institute, is gratefully acknowledged.

GUNTHER L. EICHHORN

JoAN F. CAIRNS

Department of Chemistry,

Georgetown University, Washington 7, D.C.

${ }^{2}$ Tuppy, H., and Bodo, G., Momatsh., 85, 807, 1024, 1182 (1954). Tuppy, H., and Paleus, S., Acta Chem. Scand., 8, 353 (1955). Theorell, H., and Akesson, A., J.A A mer. Chem. Soc., 63, 1818 (1941).
Margoliash, E., Nature, 175, 293 (1955).

-Theorell, H., Biochem. Z., 298, 242 (1938); 301, 201 (1939).

- Dwyer, F. P., and Gyarfas, E. C., Nature, 163, 918 (1949); Chem. Rev., 54, 9.59 (1954).

5 Glauser, S., and George, P. (private communication: The Purification is a modiffcation of the method of Margoliash, E., Biochem. J., 56, 529 (1954)).

'Theorell, H., Biochem. Z., 285, 207 (1936).

\section{Isolation of a Glycine-Carbohydrate Residue from Muscle Collagen}

ONE of a group of related, macromolecular sub. stances composed of an amino-acid linked to $\mathbf{a}$ polysaccharide has been isolated and partially characterized. 'This group of substances, obtained by means of proteolysis of muscle collagen by carboxy. peptidase and subsequent electrophoresis of the non-dialysable fraction, suggests the existence of a polysaccharide residue linked to the C-terminal amino-acid.

Collagen was prepared from rat muscle by a com bination of the methods described by Neuberger et al. ${ }^{3}$ and by Fitch et al. ${ }^{2}$, employed in the order stated. The collagen preparation used in these experiments was obtained from rats which had been injected intraperitoneally with eight doses of glycine-2-14 C (total counts per min. injected, $6.35 \times 10^{6}$; specific carbon-14 activity, $1.88 \times 10^{2}$ c.p.m./mgm.). The rats, which were killed $56 \mathrm{hr}$. after the injection of the last carbon-14 dose, were also used in related studies reported elsewhere ${ }^{3}$. Similar experiments using D-glucose-U- ${ }^{14} \mathrm{C}$ will not be described here in detail.

In a typical experiment, $50 \mathrm{mgm}$. of muscle collagen was incubated at $37^{\circ} \mathrm{C}$. for $20 \mathrm{hr}$. with $4 \mathrm{mgm}$. of thrice recrystallized carboxypeptidase in the presence 